\title{
TRANSATLANTIC RADIO COMMUNICATION
}

\author{
BY E. F. W. ALEXANDERSON
}

\section{Abstract of Paper}

The paper defines the state of the art of today which is the result of developments during the war. Transatlantic radio communication is at present maintained by five first class stations, two in America and three in Europe. These stations operate at wave-lengths between 12,500 and 17,000 meters. The range of wave-lengths suited for such traffic is rather limited, the desirable wave length being included between the limits of 10,000 to 20,000 meters. New developments indicate three methods for increasing the radio traffic without interference between the different messages. These methods are increase of the transmitting speed, closer spacing of wave lengths and directive reception. If these technical possibilities are intelligently used, the author predicts that radio communication will be equal to all demands that will be placed upon it.

The second part of the paper describes the radio transmitting system for the development of which the author is responsible. This system is represented by the naval radio station, New Brunswick, N. J. and comprises new means for generating modulating, and radiating the continuous wave energy. The generator is the high-frequency alternator with which the author's name has become associated. The modulating system is the "magnetic amplifier" which is described in a paper by the author before the Institute of Radio Engineers. The "multiple antenna" system of radiation is described in this paper for the first time. The general theory and figures for the increased radiation efficiency are given. The author also predicts that the multiple antenna will make possible directive radiation on a large scale.

IT has already become generally known that a new highway for world traffic has been opened up through the development of transatlantic radio communication. It is now a matter of history that radio was largely used for communication between the United States and armies in Europe and that the Great War was brought to a close by negotiations conducted by radio which led to the armistice. Now, we are ready for an international commerce of unprecedented scope, but lack adequate means for communication.

The recent achievements of radio technique have become common knowledge, and the world has now turned to this new method of communication clamouring that it step in and save

\footnotetext{
Manuscript of this paper was received September 6, 1919.
} 
the day. This is a condition which places a serious responsibility upon radio engineers. Fortunately, the technique has emerged from the cloud of mystery that used to surround it, and we are in position to treat the problem cooly and scientifically like any other problem in electrical engineering. However, it must not be inferred that the task is any easy one, if the radio technique is to fulfill all the hopes which are placed on it.

It has been demonstrated during the war period that transoceanic communication has become thoroughly reliable, every day in the year, and practically every hour in the day. Thus far, we can say, that the problem is solved. But a second question will be raised: What volume of traffic can be carried by the means at our disposal at the present time, and what is the relation of this radio traffic to the world traffic of to-day and to the world traffic of the future? The facts of the case are briefly the following:

Experience has shown that the wave lengths which are most suited for transoceanic communication lie between 12,000 and 17,000 meters. "This space in the ether" has already been taken up by five first class transmitting stations which, during the war period and up to the present time, have been in continuous service for transatlantic communication. Of these stations, two are in the United States, one in England, one in France and one in Germany. By extending the range of wave lengths down to 10,000 and up to 20,000 meters, and following the same system of intervals there would be room for about seven more stations or a total of twelve first class transmitting stations.

A first class station has such radiating power that its messages can be received in all parts of the world. This is one of the advantages of radio communication; but it implies that if such a station is to be used to full advantage it must have a "right of way" for its wave length over the whole world. Thus if we look at the matter pessimistically, without allowance for the improvements that further engineering developments are likely to bring, it would look as if the capacity of the world for first class radio stations would be about twelve. The rate of transmission at the present time from these stations is about twenty words a minute and it would thus be easy to figure out that the capacity of radio for handling any considerable portion of world communication would be totally inadequate. 
The other side of the picture, which the radio engineer of the future must study carefully and closely, should indicate the technical possibilities for improving the situation. The tendency of present day developments points to the following means for expansion of radio traffic:

1. Increase in speed of transmission.

2. Improved selectivity based on the direction of the wave.

3. Improved selectivity making possible closer spacing of wave lengths.

As a basis of discussing the general situation it may be here stated as simple facts:

1. That signals have been transmitted and received at considerably more than 100 words a minute.

2. That signals have been received from Europe while an American high power station within comparatively short distance was radiating on the same wave length.

3. That is has proved practical to separate radio signals differing in wave length considerably less than 1 per cent.

Based on these facts, it is probable that the transmitting speed in the future will average 100 words a minute instead of 20 words a minute.

That the selectivity for direction of the waves will multiply by five the number of stations that may be operated on one wave length; and that the selectivity with reference to wave length will be improved so that the wave lengths of messages will be within 1 per cent of each other, instead of 7 per cent which is the spacing of the stations at present.

These prospects, taken in combination, give us an optimistic picture in which the possible capacity for transoceanic radio traffic of the world is 175 times as great as it is with the practise of to-day.

To claim that the traffic capacity could immediately be increased nearly 200 times, would be an exaggeration, because the different improvements which have been made may partly conflict with the execution of each other if they are to be used simultaneously. This optimistic picture is therefore to be regarded as a goal-perhaps never to be reached, but it points the way for almost unlimited possibilities for progress by continued engineering efforts.

In order to avail ourselves of these three improvements simultaneously, the transmitted wave must be a continuous wave which does not "spill over" with harmonics, decrements or 
variations, into the range of wave length assigned for other communication. When wave lengths are spaced 1 per cent apart, each wave must be sufficiently pure so as to have na objectionable components outside the limits of one-half of one per cent. While rules to this effect should be rigidly enforced it must be appreciated that there are certain fundamental limitations.
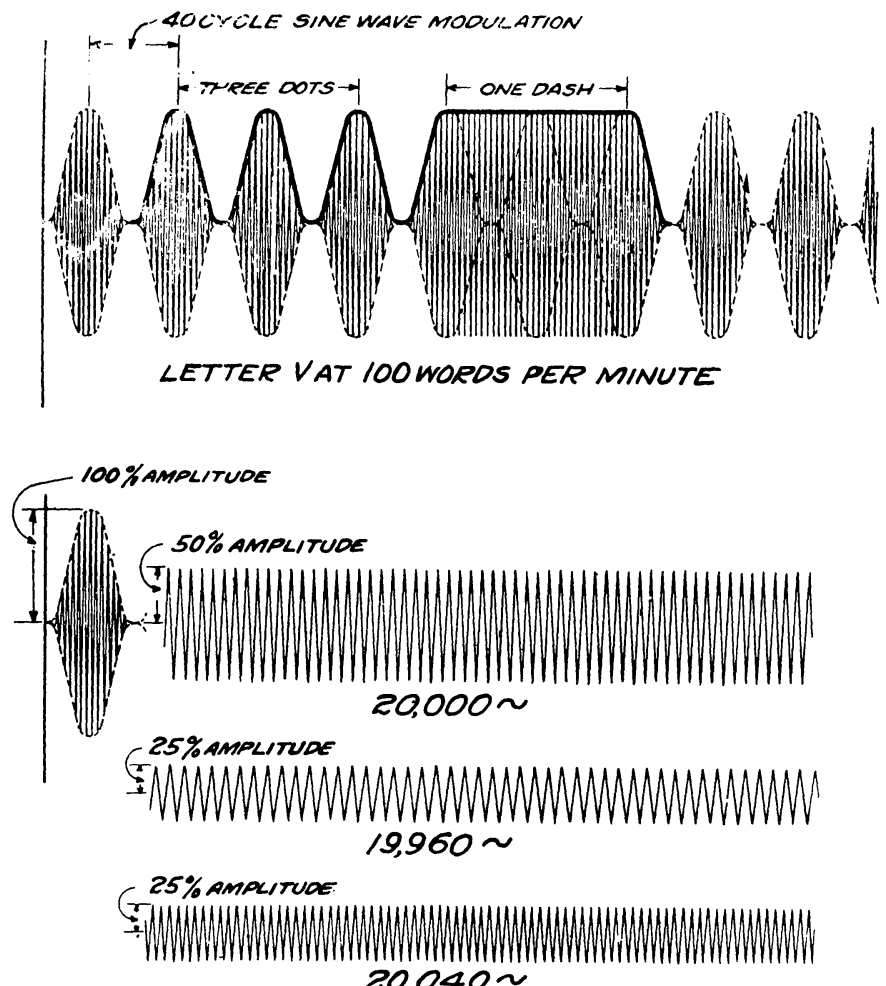

20,040

Fia. 1-Mfithod of Resolving Sine Wave Modulation into Three. Continuous Waves

\section{High Speed Signaling}

Modern transoceanic radio signaling is conducted by means of continuous waves. It must be appreciated, however, that signaling by an absolutely continuous wave would be impossible, because the making of dots and dashes introduces increments and decrements in the radiation. It will be shown that a repetition of increments and decrements can be resolved into 
a group of closely adjacent continuous waves. This agrees with the well known fact that the tuning of a wave with a decrement is known as a broad tuning. To illustrate this point we may take as a basis a signal at 100 words a minute ( 5 letters per word). If it is assumed that the increments and decrements in making the dots are not sharp interruptions but a continued variation by sine wave curves as indicated in Fig. 1, it is found by an analysis that such a wave may be resolved into a group of continuous wave components within the limits of 40 cycles above and below the average. This is the theoretical minimum width of the band of wave lengths which are necessary to transmit 100 words per minute. If the dots are defined by sharper interruptions the wave becomes still "broader" and it would not be unreasonable to say that the minimum practical band of wave lengths for 100 words per minute is 100 cycles on each side of the fundamental. This would make possible a spacing of the waves 1 per cent apart when using a wave length of approximately 15,000 meters. Messages at a higher rate of speed will occupy a correspondingly wider "space in ether."

As a conclusion from this analysis it may thus be said that an increase of the speed to 100 words a minute and increase of messages to a spacing 1 per cent apart may be accomplished simultaneously provided that waves are used of such a character and modulation that they contain no radiation except the one needed to accomplish the intended purpose.

\section{DIRECTIVE RECEPTION}

The second means for increasing radio traffic consists in improving the selectivity by taking advantage of the direction of the waves. The author's paper printed in the Proceedings of the Institute of Radio Engineers, August, 1919, described a receiver referred to as the "Barrage Receiver" which was used to demonstrate directive reception. There are several other types of receiving devices, developed by the United States Navy and other investigators, which accomplish substantially the same purpose. The broad principle underlying all these directional receiving devices is the one discovered by Bellini and Tosi and generally referred to under their names.

In the U. S. Navy's tests of the "Barrage Receiver", it was proven that it is possible to carry on simultaneous two-way communication on exactly the same wave length. If this 
method of directive reception is carried out consistently in a world system of communication, it may be assumed that transmitting stations operating on the same wave length may be located approximately as shown in the map in Fig. 2-one in Europe, one on the American east coast, one on the American west coast, one in the Far East and one in South America. The American receiving station for European signals in such a system should be located east of the American Atlantic Transmitting Station, and in line with the Pacific Transmitting Station. Thus messages from the two American transmitting stations could both be simultaneously neutralized in the American Receiving station by a "Barrage Receiver," while signals on the same wave length are received from Europe. Interference from the South American station may be neu-

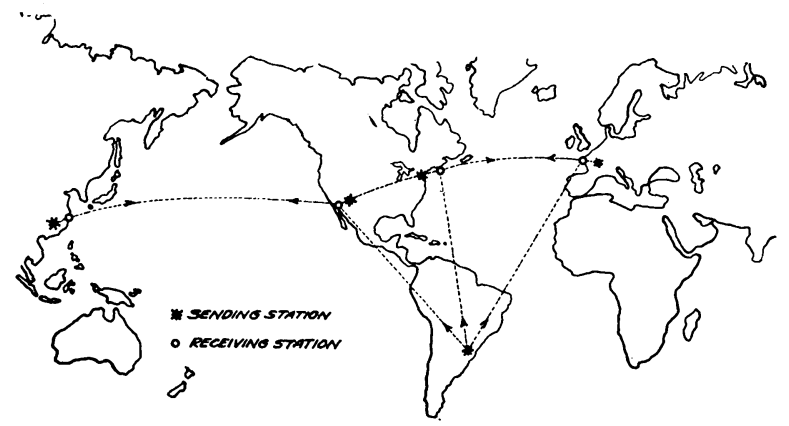

Fig. 2-Proposed Simultaneous Radio Transmissions on the Same Wave Length

tralized by the use of a double barrage system, while the station in the Far East, though it may not be exactly in line with the two others would be sufficiently near the general direction so that, considering the great distance, it would not cause interference.

If this communication system is to be duplicated on a number of other wave lengths, the practical conclusion follows that the transmitting stations as well as receiving stations for each district should be grouped in centers, and these centers located relatively so as to make the directive neutralization as effective as possible. The neutralization of several transmitting stations simultaneously may not always work out as first designed. It has been shown by investigations of the Navy Department that the radio waves do not always follow straight 
lines and not always the same path. However, discrepancies from such origin may again be overcome by further extending the principle of neutralizing waves from several directions simultaneously.

\section{Closer Spacing of Wave Lengths}

The method of increasing traffic capacity by closer spacing of wave lengths has great possibilities. It has been shown that the selectivity with reference to wave length can be greatly increased by several successive tunings in either the radio, the audio or some intermediate circuit. It is thus entirely practical to receive either by ear or by photographic records, signals

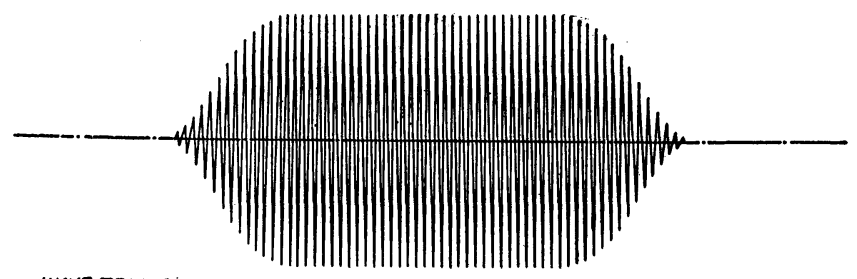

WAVE TRAN SIOWING ACTUAL NUMBER OF RADIO CYCLES IN A DOT OF THE TELEGRAPH COOE TRANSMITTEO AT THE RATE OF 500 WOROSPER MINUTE AT

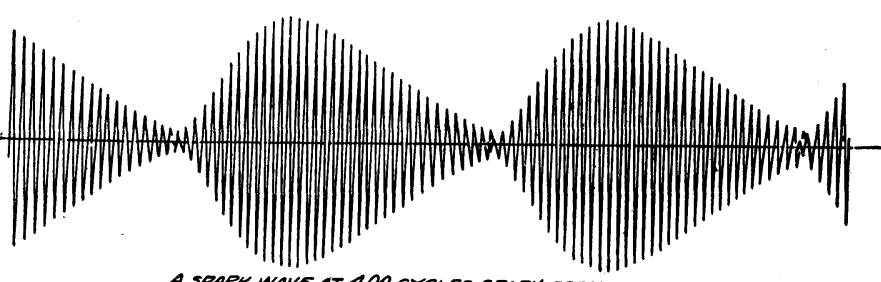

Fig. 3

which are considerably less than 100 cycles apart. The theoretical limits for such selectivity in connection with high speed transmission are defined below. As illustrated in Fig. 1, a high speed message is not a single continuous wave, but a band of wave lengths -100 words per minute occupying a space of about 200 cycles. Therefore the same degree of selectivity is not to be expected with a high-speed signal if the interfering radiation is of considerable intensity.

Speaking in terms more familiar to practical radio operators it may be said that high speed telegraph signals assume to some degree the objectionable characteristics of a spark signal. As an illustration of this, Fig. 3 shows the relative decrements in a continuous high-speed telegraph wave and a spark wave, show- 
ing that the increments and decrements of the continuous wave signal at 500 words per minute are about equal to a spark wave of 400 sparks per second. These illustrations apply to wave lengths of about 15,000 meters.

\section{The Radio Transmitting System}

Several types of radio transmitting systems are at present in use with a high degree of success. The descriptive matter in this paper will, however, be confined to the system for which the author is responsible as represented by the Naval Radio Station at New Brunswick, N. J.

Generally speaking, any radio transmitting system consists of three essential elements:

1. The generator of radio frequency energy.

2. The modulating system, whereby the energy is controlled so as to produce the dots and dashes of the telegraph code or the modulations of the human voice.

3 . The antenna or radiating system.

\section{Generating System}

There are four types of generating systems of high-frequency energy in use at the present time.

1. The spark or impulse generator.

2. The Poulsen-Arc generator.

3. The high-frequency alternator.

4. The vacuum tube oscillator.

The system which will be described, is of the type employing a high-frequency alternator. The installation of New Brunswick contains a 50-kw. alternator shown in Fig. 4 which was operated for sometime for experimental purposes with radio telephony at a wave length of 8000 meters, and later in transatlantic telegraph service at 9300 meters.

A larger equipment which has been in continuous service for the last year consists of a $200 \mathrm{kw}$. alternator shown in Fig. 5, 6 and 7. Fig. 5 shows the machine partly assembled, the rotor consisting of a solid steel disk. The spaces between the polar projections are filled with non-magnetic material so as to present a smooth surface and thereby reduce air friction to a minimum. The disk runs between the two laminated armatures which are cooled by water pipes as shown in the photograph. The armature winding which consists of wire wound back and forth in straight open slots, is divided in 64 
PLATE XL.

A. i. E. E.

VOL. XXXVIII, NO. 10

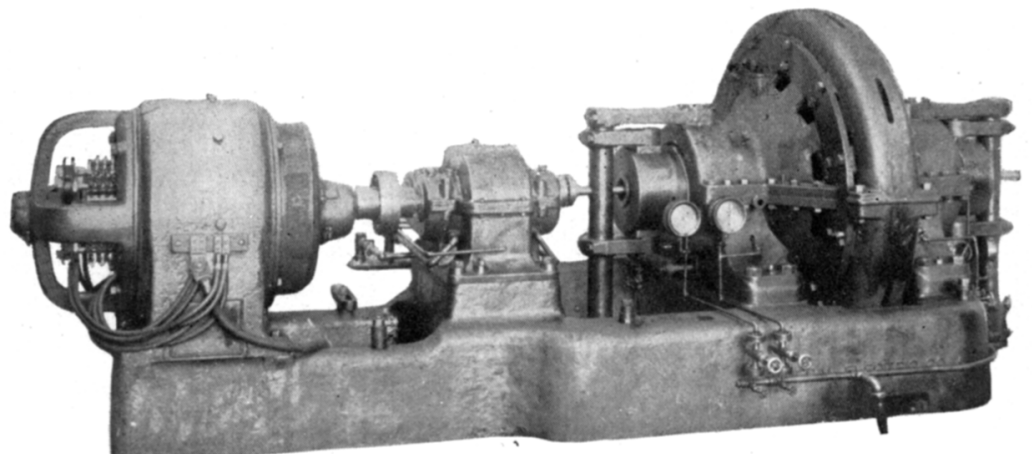

Fig. 4-50-Kw. 50,000-Crcle Altersator

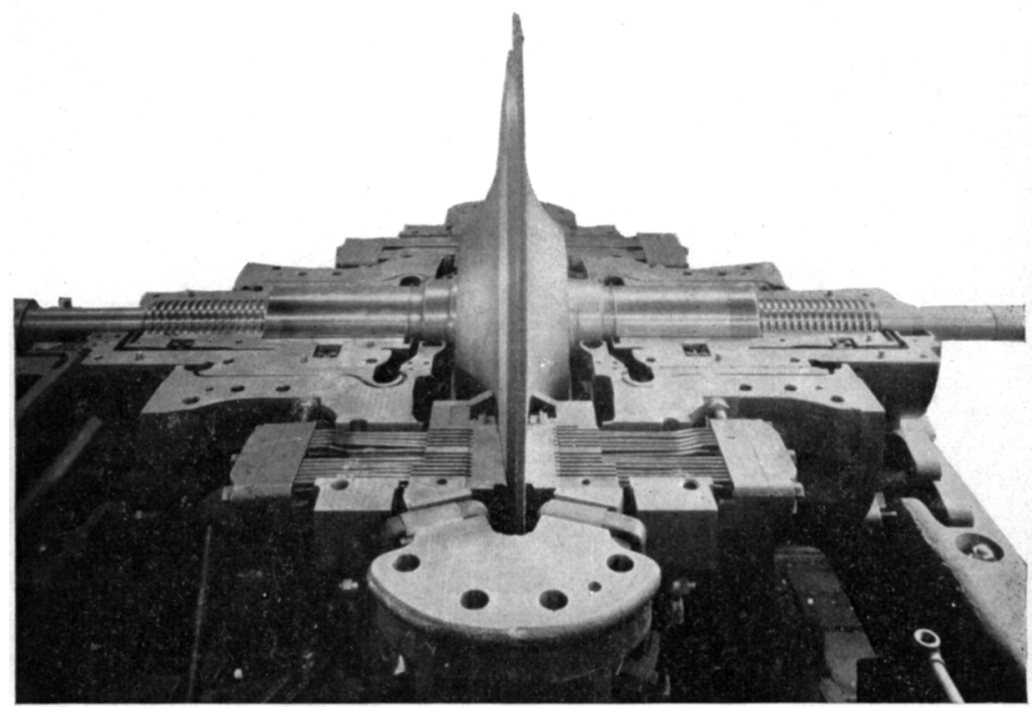

Fig. 5-200-Kw. High-Frequency Altersator

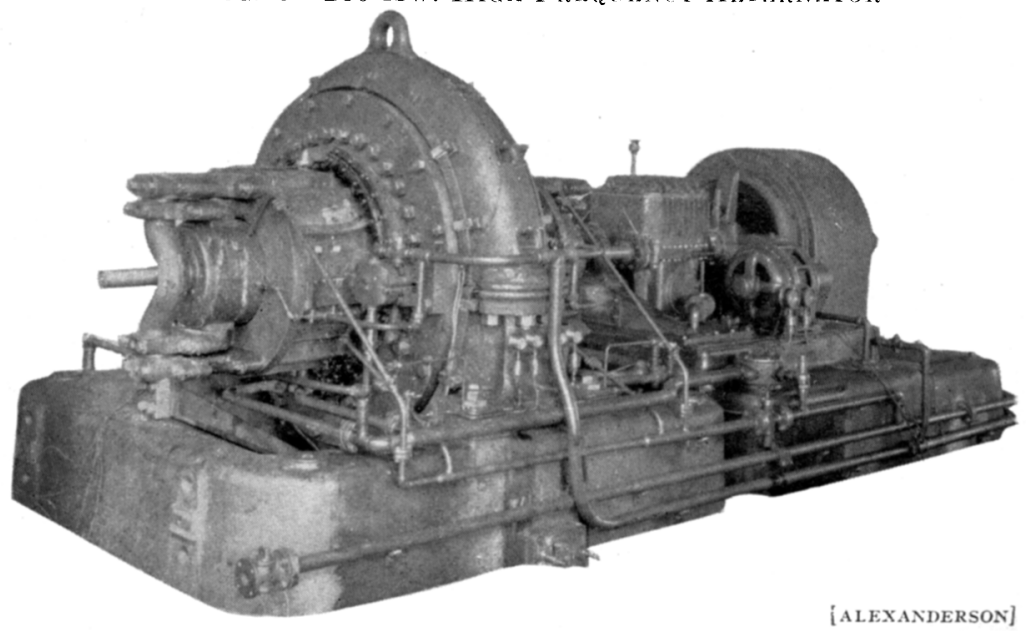

Fig. 6-200-Kw. High-Frequescy Altersator 

PLATE XLI.

A. I. E. E.

VOL. XXXVIII, NO. 10

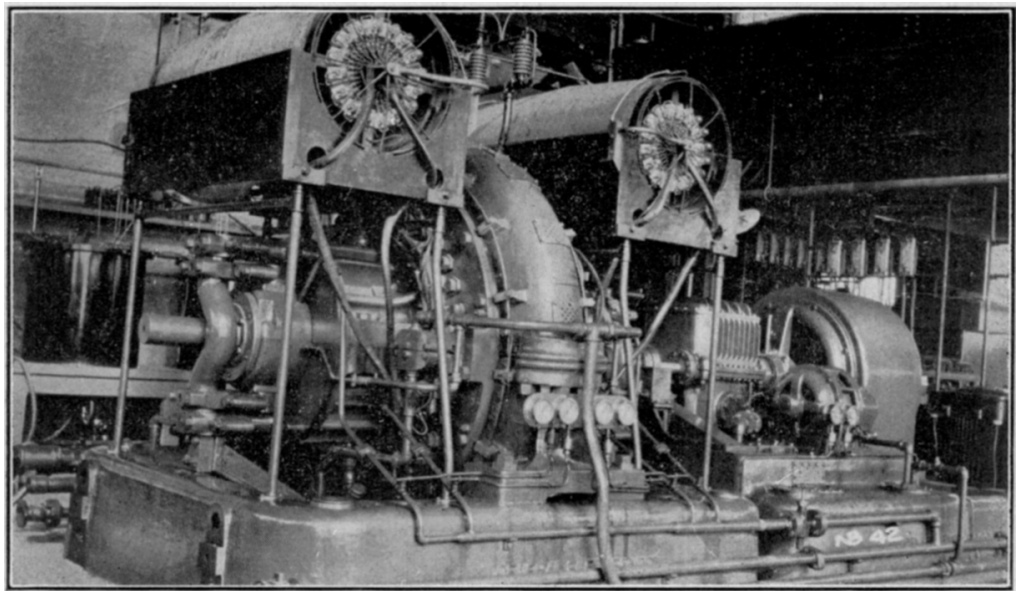

Fig. 7 -200-Kw. Alternator Set as Installed in the Naval Radio Station, New Brenswick, New Jersey

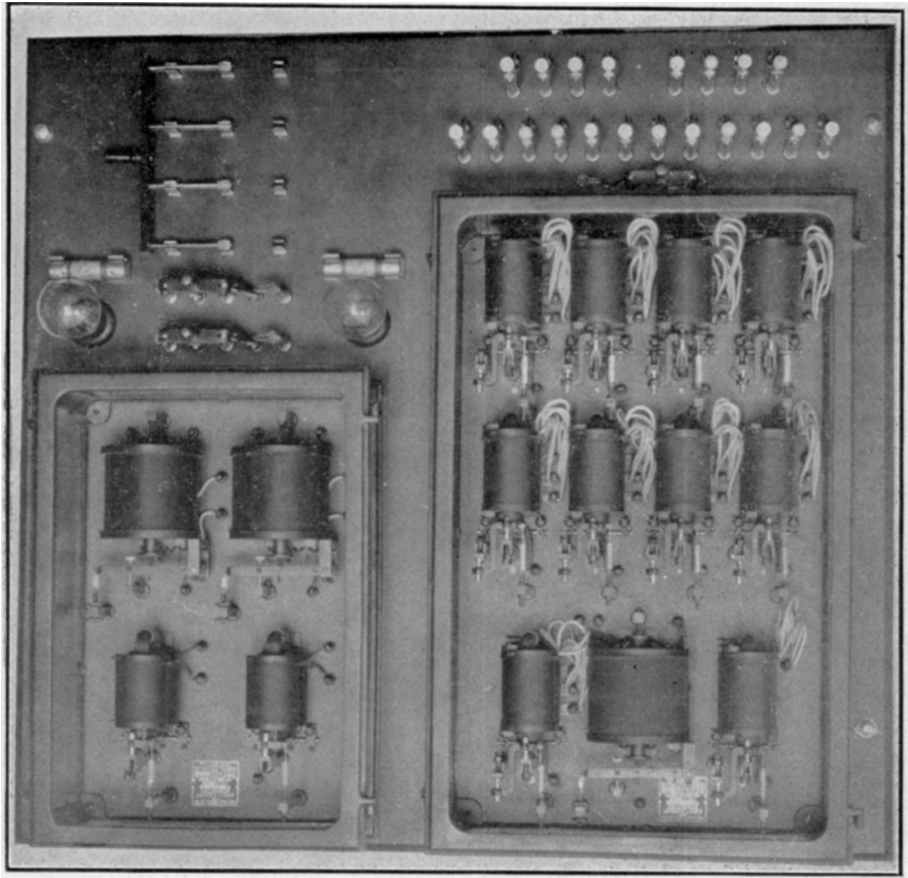

[ALEXANDERSON]

Fig. 9-Combined Current and Voltage Regleators for HighFrequency Alternator Control. 

sections, each section generating about 100 volts and 30 amperes. The current generated by these 64 windings is collected in the air core transformer mounted on the top of the machine, Fig. 7. This transformer has 64 independent primary windings corresponding to the armature windings. The single secondary winding of the transformer delivers the complete output of the alternator. This collecting transformer is thus to be considered as an integral part of the generating unit and for all purposes of calculation the characteristics of the generating unit, such as electromotive force and current, are given as delivered from this secondary winding. At full output the alternator delivers 100 amperes at an electromotive force of 2000 volts. It can thus be seen that the alternator is designed for a load resistance of $20 \mathrm{ohms}$. However, the same machine might be adapted for any other load resistance by selecting à different number of turns in the secondary of the collecting transformer. The reason why this particular machine is designed for a high voltage and low current will be given later in discussion of the new type of antenna with which it is used.

The 200-kw. alternator when operated at the New Brunswick wave length of 13,600 meters runs at a speed of 2170 rev. per min. It is driven by an induction motor through a gear of a ratio of 2.97:1 when the high frequency alternator is used as a source of radiation, the wave length is determined directly by the rotative speed of the machine. Thus, obviously, it is important that the rotative speed should be as nearly absolutely constant as it is possible to make it. An important accessory of the alternator set is therefore the speed regulator. The 50-kw. alternator set shown in Fig. 3 is driven by a directcurrent motor whereas, the $200-\mathrm{kw}$. set is driven by an induction motor of the slip ring type. The 50-kw. set was equipped with a direct-current motor because the problem of speed regulation of that type of motor is somewhat easier. Induction motors were, however, decided upon for the later types because alternating current power is more easily available in most localities.

\section{SPEed Regulator}

The speed regulator consists of a speed determining element and a power controlling element. The speed determining element is a resonant high-frequency circuit fed by one of the 64 alternator windings which is set aside for that purpose. The oscillating energy of this high-frequency circuit is associated 
by magnetic couplings with a rectifying circuit in which the high-frequency energy is changed into direct eurrent. This rectified current in turn, actuates the controlling magnet of a vibrating regulator of the type that is generally used for voltage regulation in power stations. When the driving motor is a direct-current motor it is easy to see how this vibrating regulator may be made to control the speed by regulating the voltage of the power supply to the motor. In order to accomplish the same object with an induction motor some new features have been introduced.

An ordinary induction motor is operated at constant potential. When the motor runs light it draws from the line a magnetizing current which is almost wattless. Thus it operates at a low power factor. When the motor is fully loaded it draws power at a high power factor-a motor of the type used having a power factor of 90 per cent.

When the New Brunswick station was adjusted for operation it was found that a wave length was desired which required the induction motor to work at 19 per cent slip. The rheostat in the secondary of the motor could easily be adjusted so that the motor would deliver the desired power with full load at 19 per cent slip. However, inasmuch, as the output of the alternator varies continually with the making of dots and dashes of the telegraph code, the motor is alternately loaded and not loaded. The tendency would therefore be for the motor to speed up during the intervals. If the potential of the power supply to an induction motor is varied, the motor torque varies by the square of the voltage. It is furthermore easy to show, by the theory of the induction motor, that if a motor consumes power at 90 per cent power factor at full load, and the load is reduced to $1 / 4$ by the reduction of voltage to $1 / 2$, the power factor will remain 90 per cent. In fact it will always consume power at 90 per cent power factor regardless of its load if the voltage supply is adjusted accordingly, so long as the secondary resistance remains constant and the speed remains constant.

Thus it may be said that the standard method of operating an induction motor is at constant potential and variable power factor. The method of operating the driving motor of the radio set may on the other hand be characterized as variable potential and constant power factor.

The problem which thus presented itself was to find means 
for varying the applied voltage in accordance with action of the speed-determining element, and this has been done in the following way:

Between the motor and the power supply is introduced a choke coil with iron core, the permeability of which can be varied by saturation. The change in permeability is produced by a direct current which is controlled by a vibrating regulator. When the motor carries full load the iron core is saturated so that the choking effect is practically zero. At fractional load the choking effect is automatically adjusted by the regulator so that the motor delivers at all times the power required to hold constant speed. The motor itself operates at all times at its maximum efficiency and power factor, but the power factor of the current drawn from the line varies with the load. Thus

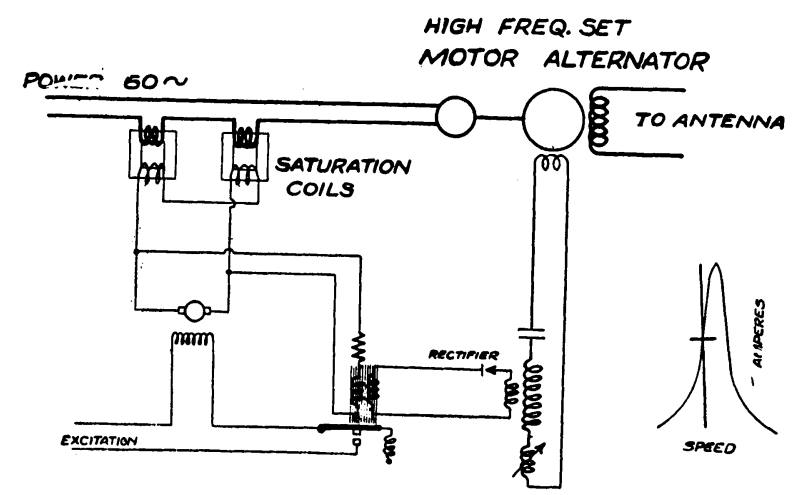

Fig. 8-Speed Regulator Alternating Current Drive

when the motor operates at $1 / 4$ load the power factor of the line is 45 per cent while the power factor of the motor is 90 per cent. The circuits of the regulator are shown in Fig. 8 and the vibrator regulator in Fig. 9.

\section{Modulating System}

The method of controlling high-frequency energy involves an apparatus which has become known as the "magnetic amplifier." This device is described in a paper by the author in the Institute of Radio Engineers, January, 1916, and therefore needs to be referred to only briefly. The magnetic amplifier is a device which is physically of the nature of an oil cooled transformer. The iron core which is made of fine laminations, is designed in such a way that the magnetic permeability of 
the iron core can be varied by magnetic saturation. By a special combination of tuned circuits as shown in Fig. 10 it has become possible to separate the controlling current from the high-frequency current so that a comparatively weak current of a few amperes controls as many hundreds of amperes in the antenna. When the transmitting station is used for telegraphy the magnetic amplifier is controlled by the telegraph relays which are a part of the wire telegraph system. During the war service, the telegraph key was operated in the centralized operating room of the Naval Communication Department in Washington. When the station is used for telephony the controlling current is an amplified telephone current.

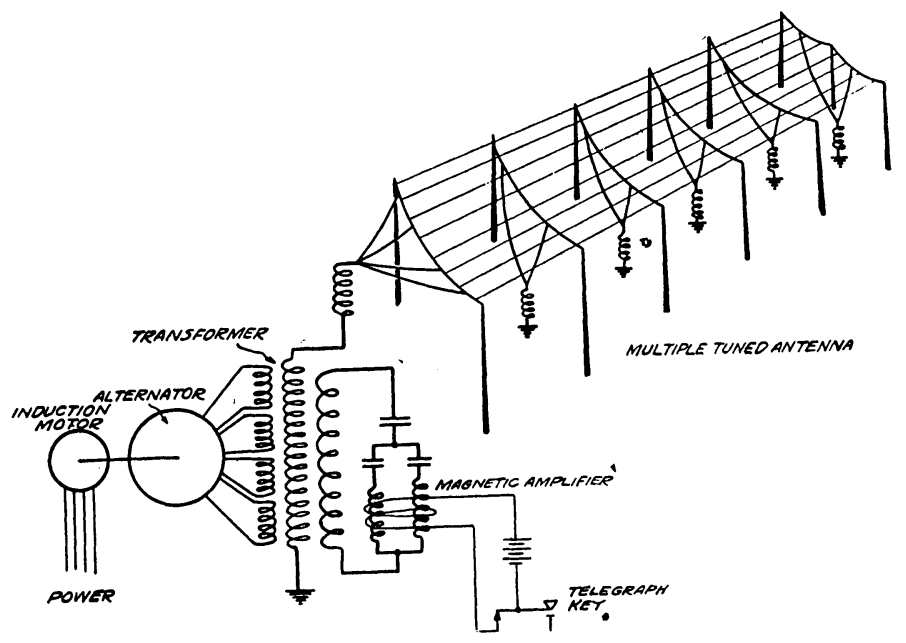

Fig. 10

While the magnetic amplifier has proven to be a very satisfactory and reliable controlling device for ordinary telegraphy its particular advantages are most prominent in high-speed telegraphic transmission and telephonic transmission, on account of its instantaneous magnetic action without any arcing contacts. Fig. 11 shows an oscillogram of radiation at 100 words per minute and a copy of a photographic record of reception at the same speed. Fig. 12 shows the telephone modulation of the antenna current when Secretary Daniels was speaking over the telephone line from Washington, controlling the output from the New Brunswick station, thereby transmitting his voice to President Wilson's ship at sea. 
PLATE XLH

A. I. E. E.

VOL. $X X X Y I I$, NO 10

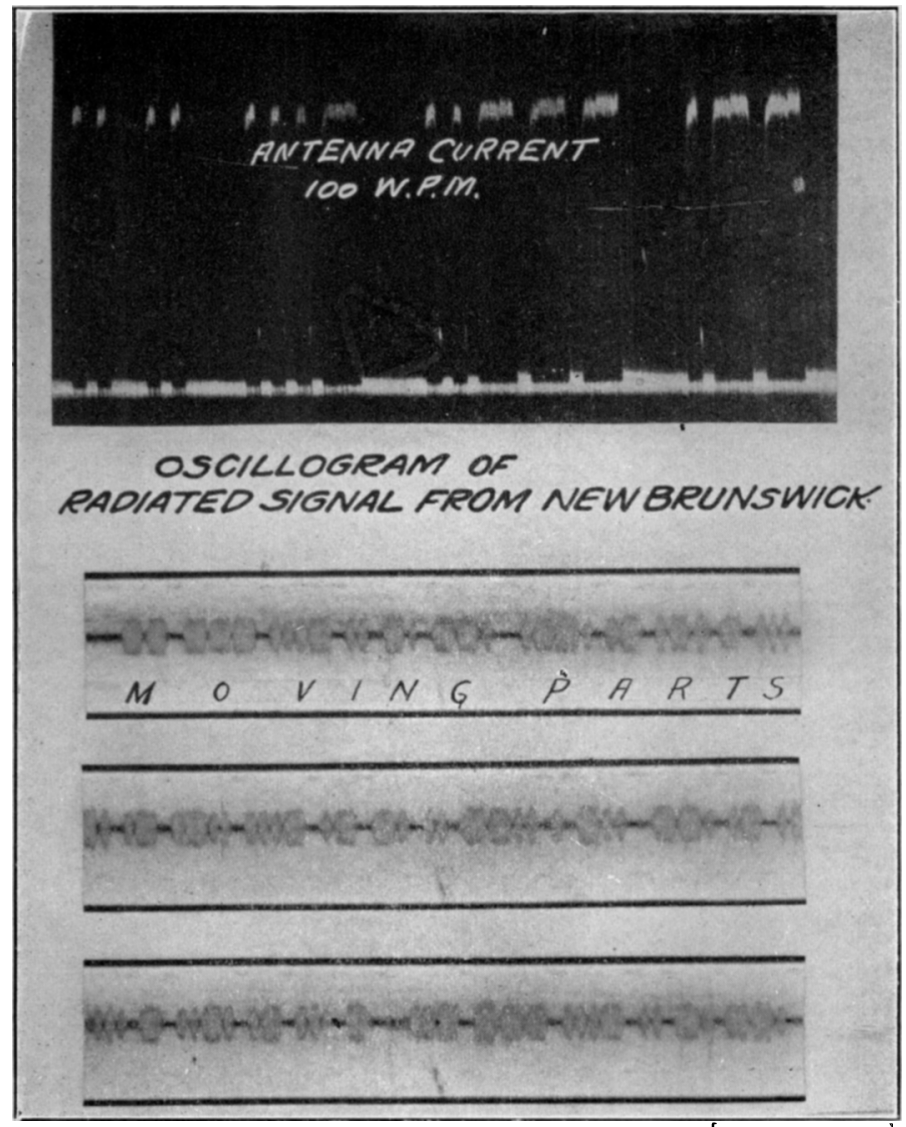

[ALEXANDERSON]

Fig. 11-Photograph of Received Signal from New Brtnswick at 100 Words per Minute 



\section{The .Multiple Antenna}

The antenna of the New Brunswick station represents a new departure in the method of radiation. The old antenna structure was originally one of the horizontal Marconi antenna, $5000 \mathrm{ft}$. (1500 m.) long, $600 \mathrm{ft}$. (180 m.) wide supported on towers $400 \mathrm{ft}$. $(120 \mathrm{~m}$.) high. The original antenna had a resistance of $3.8 \mathrm{ohms}$.

The antenna as operated now has a resistance of $0.5 \mathrm{ohms}$ distributed approximately as follows:

Radiation resistance............... $0.07 \mathrm{ohms}$

Tuning coils and insulation.......... 0.10

Ground resistance............... 0.33

Total multiple resistance........ $0.5 \mathrm{ohms}$

The reduction in total resistance of the antenna is due to the reduction of the ground resistance. While the old antenna had one tuning coil located in one end, the new antenna has six tuning coils as shown in Fig. 10 and Fig. 13.

\section{Theory of the Multiple Antenna}

The theory of the multiple antenna can be explained in several ways. Without going into details, an explanation will be presented giving the point of view which has proved most useful for general discussion. For this purpose the multiple antenna may be considered as an aggregate of several antennas of the ordinary vertical type each having its own tuning coil. When regarded in this way, the multiple antenna in New Brunswick is equivalent to six independent radiators placed $1000 \mathrm{ft}$. apart. The ground resistance of each of the radiators is 2 ohms, the coil resistance $0.6 \mathrm{ohms}$ and the radiation $0.07 \mathrm{ohms}$ making a total resistance of $2.67 \mathrm{ohms}$. Each of these radiators has an aerial $1000 \mathrm{ft}$. long and $600 \mathrm{ft}$. wide, counted at an average height of $300 \mathrm{ft}$. A total resistance of about $2.67 \mathrm{ohms}$ is, as a matter of fact, the resistance to be expected from an antenna of such dimensions and ordinary design. If one individual antenna such as described is operated with a radiation of 100 amperes, the energy consumption of the antenna would be $26.7 \mathrm{kw}$., and the radiation efficiency would be 0.07 ohm divided by $2.67 \mathrm{ohm}$ which is 2.6 per cent. If it is desired to increase the radiation from 100 amperes to 600 amperes the energy consumption would be 36 times as great that is, $960 \mathrm{kw}$. There is, however, another way to produce a 
radiation equivalent to 600 amperes. If six separate antennas of the dimensions described were built and each operated with a radiation of 100 amperes, each antenna would emit a system of waves proportional to 100 amperes radiation. If all the waves emitted by the six antennas were in phase, the amplitude of the combined wave would be six times as great as the wave emitted by one antenna. Thus the amplitude of the combined wave would be equal to the amplitude of the wave emitted by one antenna when operated at 600 amperes. The energy consumption required for operating one antenna was $26.7 \mathrm{kw}$., thus it might off-hand be concluded that the energy consumption required for operating the six antennas simultaneously would be $160 \mathrm{kw}$. This conclusion is not exactly correct because there is an interaction between the radiating effects of the different antennas resulting in a somewhat higher energy consumption. This might be expressed as follows:

The radiation resistance which is $0.07 \mathrm{ohm}$ is common for all six antennas, whereas the ground and coil resistance of 2.6 ohm belongs to the different antennas, individually. Thus the combined circuit of the multiple antenna can be represented by the common radiating resistance of $0.07 \mathrm{ohm}$ connected in series with a group of six multiple resistance of $2.6 \mathrm{ohms}$ each, so that the total current of 600 amperes flows through the 0.07 ohm radiation resistances which represent the ground and coil resistance of the individual antennas. Hence the total energy consumption of the combined antenna is found to be $180 \mathrm{kw}$. out of which $155 \mathrm{kw}$. is ground coil and insulation loss and 25 $\mathrm{kw}$. radiation. The radiation efficiency of the multiple antenna is thus 14 per cent against the radiation efficiency of 2.6 per cent for the individual antenna. The above calculation is made for the present operating wave of 13,600 meters. If on the other hand the same calculation is made for the same length of 8000 meters, which has been found more efficient for telephonic transmission, it is found that the radiation efficiency is 30 per cent, the energy being distributed as follows:

$$
\begin{aligned}
& \text { Radiation resistance........ } 0.2 \text { ohms } \\
& \text { Coil and insulation .......... 0.07 } \\
& \text { Ground resistance........... . . 0.33 } \\
& \text { Total multiple resistance....0.6 ohms }
\end{aligned}
$$

For the sake of convenience in dealing with the radiation from a multiple antenna it has become the practise to indicate 
the total radiation from the multiple antenna as the sum of the currents measured in the six ground connections. The equivalent multiple resistance of the antenna is then determined by the equation $I^{2} R=$ energy consumption, where $I$ is the sum of the currents in the ground connection.

It has been assumed above that each of the individual antennas is operated so that the waves sent out by the same are not only of the same frequency, but exactly of the same phase. It remains to be shown how this is accomplished. Fig. 13 shows the relation between the antenna and the multiple tuning coils and the alternator. As shown by the diagram, the al-
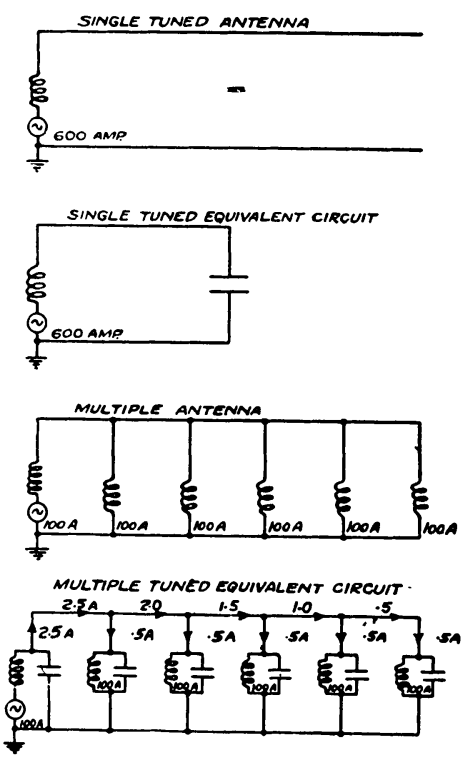

FIG. 13

ternator is connected in series with one of the six tuning coils. Arrows on the diagram indicate further how the oscillating currents and the energy currents are distributed. Six independent oscillating circuits are formed, the current in each ground connection corresponding to the charging current of the corresponding section of the aerial. If the antenna had been shock excited so that it continued to oscillate in the way indicated, no current would flow in the horizontal wires between the different sections of the antenna. However, in order to maintain continued oscillations a flow of energy must take place from the alternator which is connected to one of 
the six tuning coils. When the antenna is operated with 100 amperes in each of the ground connections, the energy consumption as shown above is $180 \mathrm{kw}$., that is, each oscillating circuit consumes $30 \mathrm{kw}$.

What actually takes place is the following: The tuning coil to which the alternator is connected transforms the energy of the alternator into a power supply at a potential of 60,000 volts, and each of the oscillating circuits draws energy from this power supply at that voltage. Thus the energy currents consumed by each oscillating circuit is only 0.5 amperes. It can thus be seen that while the total oscillating current of the antenna is 600 amperes the energy current which flows horizontally from the power source to the multiple oscillating circuits is only a total of 2.5 amperes. In other words, the energy which is delivered by the first tuning coil in the form of 100 amperes at 1800 volts is transformed by the first oscillating circuit and distributed as in a transmission line from which 0.5 amperes at 60,000 volts is drawn in five places. The analogy between the multiple antenna and a high-tension power distribution system is thus apparent.

This point of view is a departure from the conventional theory of radiation; but it must be remembered that there was a time in the development of electric power technique when the introduction of the high-tension multiple distribution system was a radical departure.

\section{DiRECTIVE RADIATION}

The multiple antenna as described in its simplest form is adjusted so that the radiation from each of the individual oscillators is in phase. If, however, the antenna dimensions are so chosen that the phase displacement of the traveling wave between the different radiators becomes an essential factor, it is possible to obtain directive radiation. The radiated wave then will not be a simple circular wave but an interference pattern which may be treated like the corresponding phenomena in light and sound waves. Furthermore the phase displacement of the oscillations of the individual radiators may be regulated by tuning. Thus a variety of interference patterns may be created and analysis of these possibilities shows that an efficient unidirectional radiation by such methods should be possible.

Methods for unidirectional radiation have been established 
PLATE XLIII.

A. I. E. E

VOL. $X X X V I I I, N O .10$

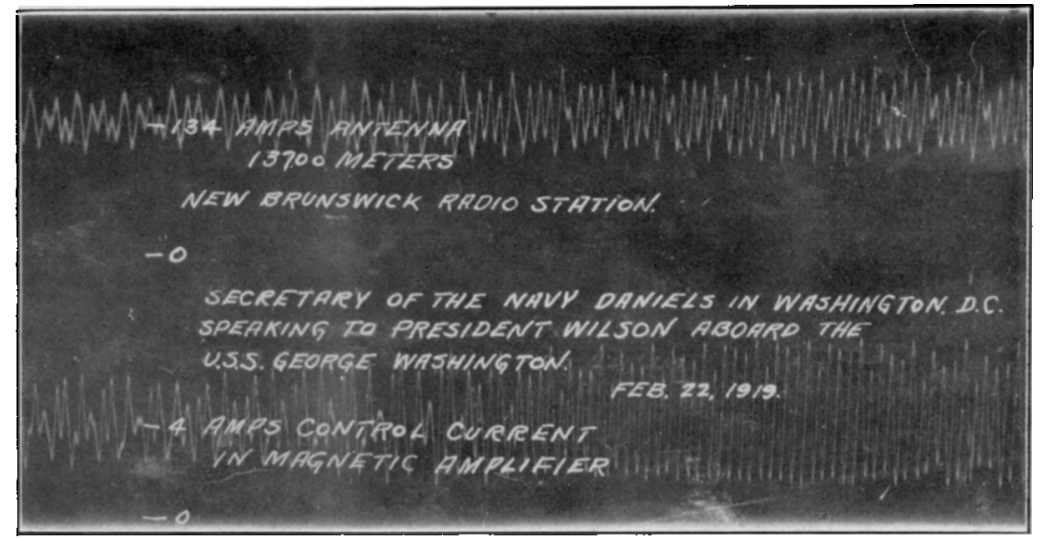

Frg. 12

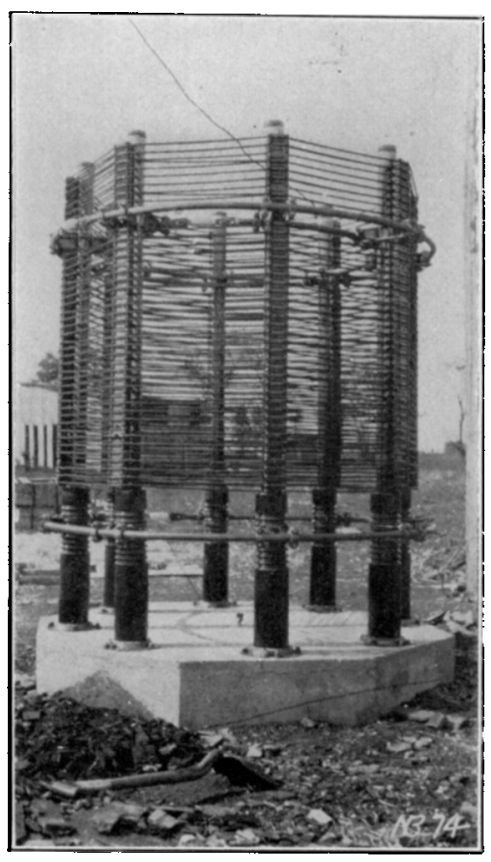

Fig. 14-Outdoor Tuning CoIL

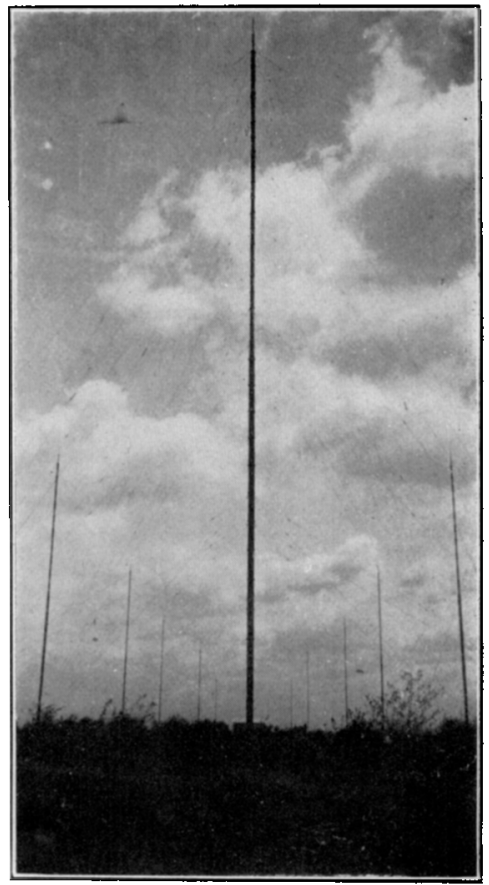

[ALEXANDERSON] Fig. 15-Perspective of Antenna 

through the well known work of Bellini and Tosi. Through the courtesy of Mr. Bouthillon of the French Post Office, results of tests made in France have been placed at the disposal of the author, which show conclusively directive radiation by the Bellini and Tosi antenna.

With the dimensions of antennas used up to the present time, efficient directive radiation has not been practical. It has, however, been proved by various tests that the system of a central power source and a distribution system of energy to a large number of multiple radiators places at our disposal means for constructing radiators of dimensions of one wave length or more. The New Brunswick antenna (1500 meters long) has a minimum wave length of 8000 meters as a single antenna, whereas it can be operated as a multiple antenna at 2000 meters wave length. A detailed analysis of the possibilities of multiple radiation would fall outside the scope of this paper but the author is in position to predict with confidence that directive radiation on a large scale will not only prove practical but that it will be the most efficient method of radiation.

To add directive radiation to the proposed program for increasing the capacity of radio traffic would perhaps be premature until it has been demonstrated on a large scale. However, it deserves mention in order to show that new principles which may be utilized for still greater expansion of the radio technique have not yet been exhausted. 